\title{
Radiation and risk: Is it time for a regulatory threshold dose?
}

\author{
Antony M. Hooker
}

Published online: 21 September 2011

(C) Australasian College of Physical Scientists and Engineers in Medicine 2011

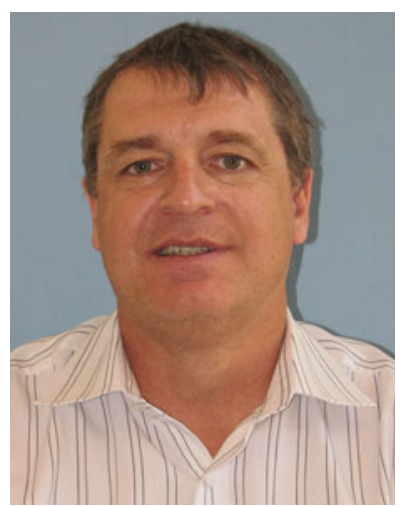

This editorial follows somewhat from Madhava Bhat's guest editorial published in the Sept 2010 issue entitled " "Fear of radiation" is frightening" [1]. As Madhava suggests, there is a heightened level of fear in the general community regarding radiation, and this is not just confined to ionising radiation (IR) but also non-ionising radiation (NIR), such as electromagnetic fields and mobile phones. I am not going to discuss NIR here except to say that I believe this area also has some worrying developments from a regulatory perspective, and in particular the use of the precautionary principle where there is a very limited scientific evidence of a biological effect.

It is understandable why the general community fears radiation. For instance, you cannot see it or feel it, but due to the sensitivity of detectors we can measure the tiniest amount of it. Over the last 60 years or so, we have been bombarded with images of atomic bombs, people in

\footnotetext{
A. M. Hooker $(\bowtie)$

Faculty of Health Sciences, School of Medicine,

Flinders University, Adelaide, SA 5042, Australia

e-mail: tony.hooker@epa.sa.gov.au
}

"radiation suits" and pictures of the known damaging effects of high dose radiation. In addition, images of Homer Simpson "working" in the Springfield nuclear power plant, and the subliminal messages found in many of our comic books suggest that radiation will mutate you into a superhero or villain. Currently, there approximately 221 comic characters who's powers originated from radiation [2].

As well as the images, there are many conflicting reports or opinions regarding the biological effects of radiation and these are often based on either some ideological or political belief or differences within the scientific and regulatory community itself. This situation is not helped by the current media cycle of unbalanced reporting; often with farleft or far-right political views getting more publicity due to their controversy rather than the facts. As a radiation regulator, I often encounter people who are unaware that they are exposed to background levels of radiation from cosmic, terrestrial and internal (body) radiation sources. Many also don't know that life has evolved on Earth in which natural background radiation levels some 3.5 billion years ago were about three times higher than they are now [3]. For some reason scientists and regulators have failed to remind the general community, or have been unable to get the message across, about the basics of radiation and that it's a natural phenomenon. Although the population is "bathed" in background radiation, the fact remains that most will only ever receive a small additional radiation dose from the activities they undertake or the medical procedures they require. The only time a person is likely to be exposed to high or dangerous doses of radiation is when they are undergoing medical treatment e.g. radiotherapy or involved in a radiation incident. However, what is deemed "dangerous" depends on what side of the Linear No Threshold (LNT) fence you sit on! 
The LNT hypothesis of radiation regulation assumes that any dose of radiation, no matter how small, is harmful and potentially carcinogenic. According to the LNT hypothesis, if a 1 Gy dose gives a cancer induction risk of $\mathrm{R}$, then the risk from a $0.01 \mathrm{mGy}$ dose is $\mathrm{R} /\left(1 \times 10^{5}\right)$, and so on. Thus the cancer risk can never be zero. According to the International Commission on Radiological Protection (ICRP), the cancer induction risk (R) for the whole population is $5.5 \%$ per Sv [4]. Therefore, the LNT hypothesis gives governments a reason to regulate and has been internationally accepted as the model of choice.

There has been considerable debate on the validity of the LNT hypothesis in recent years especially with the huge number of scientific peer-reviewed publications regarding radiation hormesis. Vaiserman [5], in his review of radiation hormesis reports that over 3000 publications have shown health benefits from increased exposure to IR. This scientific evidence has led to a number of groups such as the Health Physics Society (US) [6], the American Nuclear Society [7], the French Academy of Science and National Academy of Medicine [8] strongly condemning the LNT hypothesis. Others such as the National Council on Radiation Protection (NCRP) have been much slower to adopt a position on the LNT hypothesis although the NCRP Publication 136 stated "note that the cancer rates in most populations exposed to low level radiation have not been found to be detectably increased, and in most cases the rates appear to be decreased" [9]. Yet, even though there is a large body of scientific literature published refuting the LNT hypothesis, it is still the preferred radiation protection model and has resulted in the "as low as reasonably achievable" (ALARA) principle. Allison [10] suggests that what may have started as advice and guidance under the ALARA principle has now become frozen inflexibility into legislation and regulation.

As a regulator, there are advantages to the LNT hypothesis. It is easy to understand across all sectors (government, business and the public), and it's relatively easy to implement for regulation. However, should a flawed system be used just because it's easy? The LNT hypothesis has a profound psychological effect on the general community because it is stated that all radiation is harmful and carcinogenic. In addition, this type of regulation imposes large capital and operational costs onto both government and business. So if the LNT hypothesis is flawed what model should be used? This is the difficult question and one that is too complex to answer fully here due to the nature of the different types of radiation, radiation dose and radiation dose-rate but it is apparent that research studying the biological mechanisms involved in low dose radiation exposure and the phenomena surrounding radiation hormesis will provide the data to develop that model.
As stated above there have been over 3000 reports which demonstrate health benefits from increased exposure to IR, and this is especially evident in the area of low-dose, linearenergy transfer radiation. Both experimental and epidemiological evidence support hormetic-type, dose response relationships with low doses and low dose rates being protective whilst high dose radiation increases harm. There are numerous epidemiological papers demonstrating that low doses of radiation can increase longevity in humans by reducing cancer incidence as well as scientific studies of longevity in rodents pre-disposed to cancer. Research, including my own, suggests that low dose radiation stimulates adaptive and/or protective mechanisms which can prevent spontaneous/induced mutations arising and could also be responsible for the selective removal of pre-cancerous cells. Finally, there are also a number of current studies using animal models to look into the effectiveness of low dose rate radiation for the treatment of cancer.

From the literature, there has never been an excess in cancer detected for doses of less than $100 \mathrm{mSv}$. This is likely due to the small numbers of cancers induced by radiation which cannot be observed above the background cancer rate. For example, according to the Biological Effects of Ionising Radiation Committee V (BEIR V), the average risk of cancer death is approx $0.08 \%$ per $10 \mathrm{mSv}$ for acute radiation exposure across all ages, all genders and all types of cancer and approximately $0.04 \%$ for chronic exposure [11]. However, the average lifetime risk of dying from cancer not associated with radiation is approximately $30 \%$, thus the small increase in cancer death that may be attributed to radiation is impossible to see. For example, if you were exposed to a $10 \mathrm{mSv}$ acute radiation dose you would have a $30.08 \%$ chance of dying from cancer.

This brings me to the more ethical question of "how much risk as a society are we prepared to accept?" It is not possible to have a risk-free society. In our everyday lives we accept risk at some level, whether we smoke cigarettes, drive a car, drink alcohol, eat fattening foods or work in challenging and dangerous occupations. Many of these activities have far greater risks than the risk we would be exposed to from radiation, yet society is far more cautious about the latter. I would suggest that most of the general population would not attribute the same relative risk of death of a $100 \mathrm{mSv}$ dose of radiation to driving a car $64 \mathrm{~km}$. The lack of understanding around the issues of risk can also be highlighted with many of the recent media reports regarding the radiological incident at Fukushima. Although the Fukushima nuclear accident is a very serious situation, based on the 2 June 2011 International Atomic Energy Agency (IAEA) briefing, the gamma dose rates were at background for the majority of prefectures, the radionuclide deposition had also decreased and many of the restrictions on food and water had been lifted [12]. These 
results reflect the early and effective intervention of the Japanese authorities. To my knowledge there have been no media reports putting the doses of radiation surrounding Fukushima into perspective with current background rates of radiation and to risks normally encountered within the population. At this stage from the scientific evidence, it appears likely that the general Japanese population will be exposed to much less radiation than the ICRP limit for a member of public $(1 \mathrm{mSv})$ and that few will possibly be between $1 \mathrm{mSv}$ and the occupational limit of a radiation worker $(20 \mathrm{mSv})$.

Ideally it shouldn't matter what model is used. Regardless of whether it's the LNT hypothesis or a hormetic model, either could be used to estimate the risk in comparison to many of the other things we are currently exposed to in society. The challenge for governments and radiation regulators is to balance the risks with the benefits and to determine if the costs associated with the level of regulation are justified. Unfortunately in Australia the balance is not quite right at the moment. For example, what exactly is achieved by regulating airport back scatter apparatus (0.00005-0.0001 mSv), dual-energy X-radiation absorptiometry (DXA) $(\sim 0.0001 \mathrm{mSv})$ apparatus or even plain dental apparatus $(0.005-0.01 \mathrm{mSv})$ ? Surely the doses here are so low and equivalent to a few minutes to hours worth of natural background, regulation cannot be justified based on risk.

So the question remains, if a radiation dose threshold based on risk is to be used, what should it be? No doubt this will involve considerable debate. However, there are a number of potential threshold options already available to us and ones that have previously been used and studied. For example in 1951, the ICRP set the weekly radiation dose limit to $3 \mathrm{mSv}$. In 1957 the ICRP tightened this limit to $5 \mathrm{mSv} / \mathrm{yr}$ for a member of public and $50 \mathrm{mSv} / \mathrm{yr}$ for occupational exposure. These limits were in place until 1990 and since that time the occupational limit has been set at $20 \mathrm{mSv}$ for tens of thousands of people around the world who use radiation in their workplace daily. If $20 \mathrm{mSv} / \mathrm{yr}$ averaged over 5 years is considered safe or acceptable using a risk-benefit model for the lifetime of a radiation worker who spends $8 \mathrm{~h} / 5$ days/week exposed, surely this dose would be acceptable using the same risk-benefit analysis for members of public who are unlikely to exceed this dose under normal circumstances?

More recently, the French Academy of Science and National Academy of Medicine [8] reported on the evidence regarding the carcinogenic risk to radiation doses $<100 \mathrm{mSv}$ and reported that the use of LNT for assessing the risks of doses below $20 \mathrm{mSv}$ is unjustified and should be discouraged. The report further suggested that the LNT hypothesis should not be used for determining risk-benefit analyses for the more conservative dose $<10 \mathrm{mSv}$.
By implementing a low dose radiation threshold based on science and actual risk rather than fear, governments and businesses could reduce the financial and regulatory burden of radiation monitoring and assessment at doses where no detrimental health effect has been observed. However, the difficulty for governments and regulators will be how the regulation is removed and what model of regulation should be applied. For example, will it be principles-based, management-based or outcome-based regulation. I won't discuss any of the regulatory models here but as those people who have worked in the regulatory industry will know, whilst it may seem simple to suggest position statements, to make regulations to enforce those positions is not easy.

One thing is clear; as more is understood about the biological response to radiation, there will likely be scope for further relaxation of the radiation protection limits we use today.

\section{References}

1. Bhat M (2010) "Fear of radiation" is frightening. Australas Phys Eng Sci Med 33:215-217

2. Comic Vine (2011) List of radiation characters. http://www. comicvine.com/characters/?origin $=6 \&$ letter $=$ all. $\quad$ Accessed 28 August 2011

3. Jaworowski Z (1997) Radiation 3.5 billion years ago. 21st Century. Sci. Technol. 10:4

4. The 2007 Recommendations of the International Commission on Radiological Protection (2007) ICRP Publication 103. Annals of the ICRP

5. Vaiserman AM (2010) Radiation hormesis: historical perspective and implications for low-dose cancer risk. Dose-Response 8:172-191

6. Health Physics Society (1998) Radiation risk in perspective: position statement of the health physics society. In: Health physics society directory and handbook 1998-1999

7. American Nuclear Society (2001) Health effects of low-level radiation. Position statement. http://www.ans.org/pi/ps/pdfs/ps41. pdf. Accessed 28 August 2011

8. Aurengo A, Averbeck D, Bonnin A et al (2005) Dose-effect relationship and esitimation of he carcinogenic effects of low dose ionising radiation. Académie de Sciences-Académie national de Médicine. http://www.radscihealth.org/RSH/Papers/ FrenchAcadsFinal07_04_05.pdf. Accessed 28 August 2011

9. RP NC (1995) Evaluation of the linear non-threshold doseresponse model for ionising radiation. NCRP Publication 136. Bethesda, Maryland, USA

10. Allison W (2009) Radiation and reason. The impact of science on a culture of fear. Wade Allison Publishing, York, UK

11. Health Effects of Exposure to Low Levels of Ionising Radiation: BEIR V Committee on the Biological Effects of Ionizing Radiation (BEIR V), National Research Council (1990) http://www. nap.edu/catalog/1224.html. Accessed 28 August 2011

12. IAEA Fukushima Nuclear Accident Update log. http://www. iaea.org/newscenter/news/tsunamiupdate01.html. Accessed 28 August 2011 